Intervention du facteur de transcription c-Jun dans l'apoptose. Le facteur de transcription c-Jun est l'un des constituants du complexe AP1 qui se fixe à, et active des gènes impliqués dans la prolifération cellulaire et dans l'inflammation. Un complexe AP1 typique est constitué d'une sous-unité c-Jun et d'une sous-unité Fos, liées par une glissière de leucine (leucine zipper) [1]. Dans des modèles de cellules neuronales en culture, des anticorps antic-Jun, ou une protéine mutante transdominante-négative, sont capables d'inhiber l'apoptose induite par la privation de facteurs neurotrophiques [2]. Cette intervention de c-Jun dans l'apoptose est maintenant confirmée par l'équipe de Moshe Yaniv, de l'Institut Pasteur de Paris. Les chercheurs ont utilisé une protéine de fusion comportant la séquence codant pour c-Jun et le domaine de fixation de l'hormone du récepteur des œstrogènes; l'activité c-Jun de cette protéine est inhibée en l'absence d'œstrogènes et est stimulée par l'addition de ces hormones. Lorsqu'un vecteur d'expression pour cette protéine hybride est introduit dans des fibroblastes murins en culture, ceux-ci entrent en apoptose en présence d'œstrogènes, même lorsque la concentration de sérum est suffisante. L'apoptose est associée au clivage de substrats des caspases et est partiellement inhibée par la protéine antiapoptotique Bcl-2 et totalement par des inhibiteurs peptidiques des caspases de type ICE. Le domaine d'activation transcriptionnelle et la région leucine zipper de c-Jun sont indispensables à cet effet pro-apoptotique. Ce qui suggère que l'activité transcriptionnelle de c-Jun est impliquée dans sa capacité d'activer l'apoptose. La molécule pro-apoptotique Bad semble induite par l'activation de c-Jun $[3,4]$. Peut-être peut-on faire l'hypothèse que la stimulation de c-Jun sans activation concomitante de Fos aboutit à la formation d'homodimères Jun/Jun susceptibles d'activer directement ou indirectement la transcription de gènes pro-apoptotiques, par exemple Bad.

[1. Blanchard J. Med Sci 1992; 8: 455-70.]

[2. Ham J, et al. Neuron 1995; 14: 927-39.]

[3. Bossy-Wetzel E, et al. EMBO J 1997; 16: 1695-709.]

[4. De la Coste A, Perret C. Med Sci 1997; 13: 384-6.]

- Dissociation entre l'effet biologique des chimiokines et leur action protectrice contre l'infection par le VIH. Les co-récepteurs nécessaires à l'infection des cellules humaines par le VIH-1 sont maintenant connus : ils correspondent à des récepteurs de chimiokines, couplés aux protéines G. Ainsi, le CCR5 est-il le récepteur de souches de VIH-1 à tropisme pour les macrophages alors que le CXCR4 est un récepteur pour les souches établies en culture de cellules, infectant des lignées de cellules $\mathrm{T}$ et capables d'induire la formation de syncytiums $\left(m / s n^{\circ} 1\right.$, vol. 13, p. 72). Certains isolats de VIH-1 peuvent utiliser les deux récepteurs et, également, CCR3 $\left(\mathrm{m} / \mathrm{s} n^{\circ} 2\right.$, vol. 13, p. 264). Les personnes homozygotes pour une délétion partielle inactivatrice du gène CCR5 sont protégées efficacement -quoique pas totalement- contre l'infection par le VIH-1 ( $m / s n^{\circ} 8 / 9$, vol. 12, p. 1037). De plus, ex vivo, des chimiokines de type RANTES inhibent l'infection passant par CCR5, de même que SDF-1 et d'autres ligands de CXCR4 peuvent bloquer ex vivo l'infection exigeant la présence de ce récepteur. Malheureusement, il ne faut pas envisager utiliser in vivo ces chimiokines elles-mêmes, puisqu'elles sont des médiateurs extrêmement puissants de l'inflammation et donc susceptibles d'avoir de profonds effets secondaires. De plus, il était difficile, en culture de cellules, de différencier l'effet antiviral passant par une compétition pour la liaison aux co-récepteurs, de celui dépendant du signal relayé par ces récepteurs. C'est dire l'intérêt théorique et, potentiellement, pratique, des résultats publiés en avril 1997 dans la revue Science par des chercheurs de Londres, Genève et Copenhague... recherche impliquant les chercheurs de la firme Glaxo-Wellcome [1]. Cette équipe a modifié la molécule RANTES en rajoutant un radical à sa partie amino-terminale. L'AOP-RANTES ainsi obtenue n'a plus aucune action biologique de chimiokines, mais reste un excellent ligand de CCR5. En culture de cellules, la molécule AOP-RANTES se comporte comme un fort inhibiteur de l'infection de macrophages et de lymphocytes par des souches naturelles de VIH-1, actif à des concentrations nanomolaires. L'AOPRANTES peut-elle être considérée comme le premier représentant d'une nouvelle classe de médicaments potentiels du Sida?

[1. Simmons G, et al. Science 1997; 276: 276-9.]

\section{Un récepteur commun de} l'adénovirus et des coxsackievirus. Depuis 1976, on savait que des coxsackievirus de sérotype B et des adénovirus de sérotype 2 et 5 pouvaient entrer en compétition pour infecter certaines cellules, ce qui impliquait qu'ils partagent au moins un élément commun de leurs récepteurs cellulaires [1]. Cela semblait d'ailleurs d'emblée singulier tant ces deux virus sont différents, leur seul point commun étant de ne pas avoir d'enveloppe. L'adénovirus forme de grandes particules icosaédriques (c'est-à-dire, à vingt faces) et leur génome est constitué d'un ADN double-brin exprimé dans le noyau des cellules infectées. De chaque face du icosaèdre part une fibre capable de se fixer à des intégrines $\alpha_{v} \beta_{3}$ ou $\alpha_{v} \beta_{5}$. A l'inverse, les coxsackievirus sont de petites particules sphériques à peu près lisses et leur génome est constitué d'un ARN mono-brin positif. Bergelson et al. 
(Boston, MA, USA) viennent néanmoins de confirmer que ces virus, tout différents qu'ils fussent, avaient un récepteur commun qu'ils ont appelé CAR (coxsackie-adenovirus-receptor). La méthode utilisée pour cloner l'ADNc de ce récepteur est classique. A partir de cellules cancéreuses humaines en culture (cellules HeLa), les auteurs isolèrent une protéine de surface, de $46 \mathrm{kDa}$, reconnue par un anticorps monoclonal qui permettait de co-précipiter cette protéine et un coxsackievirus de type B. Une séquence protéique partielle permit de repérer un EST (expressed sequence tag) lui correspondant. Cet EST fut alors utilisé pour isoler un ADN complet de 2,4 kb codant pour une protéine de 365 acides aminés dont 222 pour le domaine extracellulaire, 107 pour le domaine intracellulaire, auxquels il faut ajouter un petit peptide signal et le domaine transmembranaire. Le domaine extracellulaire contient deux motifs de type immunoglobuline. Un vecteur d'expression commandant la synthèse de cette protéine CAR dans des cellules ovariennes de hamster chinois en culture $(\mathrm{CHO})$ confère à ces dernières la propriété d'être infectées par les deux types de virus [2]. Le récepteur à forte affinité de l'adénovirus étant recherché depuis bien longtemps, ces résultats sont manifestement importants. Cependant, tout n'est pas dit car, si l'expression du gène CAR correspond bien aux cibles connues des coxsackievirus de sérotype $B$, elle ne correspond en revanche que très imparfaitement à celles des adénovirus. En effet, le premier type de virus cause des méningo-encéphalites, des myocardites, des pancréatites... et le messager $C A R$ est particulièrement abondant dans le cerveau, le cœur et le pancréas. En revanche, des adénovirus de sérotype 2 et 5 donnent avant tout des infections respiratoires et l'utilisation de l'adénovirus comme vecteur de thérapie génique montre qu'il peut infecter une grande diversité de tissus, tels que, en-dehors du poumon, le foie, le muscle squelettique jeune, etc... Il se pourrait donc que d'autres récepteurs de l'adénovirus existent, ou bien que de très faibles niveaux de la protéine CAR, en conjonction avec les intégrines spécifiques, soient suffisants à la fixation et à l'internalisation des adénovirus.

[1. Lonberg-Holm K,et al. Nature 1976 ; $259: 679-81$.

[2. Bergelson JM, et al. Science 1997 ; 275 : 1320-3.]

\section{NEURO MED \\ PREMIÈRE CONFÉRENCE MÉDITERRANÉENNE DE NEUROSCIENCES Conférences-cours d'enseignements, posters, tables rondes, programmes d'ordinateur pour l'enseignement 3-6 septembre 1997 - Le Corum - Montpellier - France Président : Y. Ben Ari}

\section{CONFÉRENCES}

J.-P. Changeux (France), P. Strata (Italie), T. Freund (Hongrie), G. Innocenti (Suisse), M. Abeles (Israël), S. Pogun (Turquie), Y. Ben-Ari (France)

\section{COURS D'ENSEIGNEMENT}

C. Blakemore (Royaume-Uni), W. Singer (Allemagne), A. Nistri (Italie), I. Parnas (Israël), C. Hammond (France), J. Meldolesi (Italie), J. Bockaert (France), O. Kiehn (Danemark)

\section{SYMPOSIA}

Développement du SNC

L. Puelles (Espagne), P. Charnay (France), C. Goridis (France), A. Ghysen (France), R. Gallego (Espagne), M. Vidal-Sanz (Espagne)

Récepteurs du glutamate

J. Lerma (Espagne), R. Miles (France), R. Malgaroli (Italie), Y. Parnas (Isräl), M. Khrestchatisky (France), F. Nicoletti (Italie)

Trafic intracellulaire

G. Gennarini (Italie), J. Avila (Espagne), L. Ginzburg (Israël), D. Karagogeos (Grèce), A. Triller (France), L. Ferhat (France)

\section{$\mathrm{Ca}^{2+}$ et fonctions neuronales}

M. Segal (Israël), P. Mollard (France), M. Valdeolmillos (Espagne), R. Rahaminoff (Israël), M. Seagar (France), J. Nargeot (France)

Facteurs neurotrophiques

C. Henderson (France), E. Cattaneo (Italie), D. Martin-Zanca (Espagne),

P. Carrol (France), J.X. Comella (Espagne)

Épilepsie et ischémie

J. de Felipe (Espagne), S. Rivera (France), J. Valmier (France), G. Kostopoulos (Grèce), T. Dalkara (Turquie), P. Calabresi (Italie)

Oscillations et activités synchrones

E. Cherubini (Italie), F. Clarac (France), W. Buno (Espagne), Y. Yarom (Isräl), R. Khazipov (France), Y. Yaari (Israël)

Autres

M. Raiteri (Italie), E. Barkai (Israël), G. Pellegrini (Italie), A. Ambrosini (Italie)

Sous l'égide des sociétés des neurosciences de France, de Grèce, d'Isräl, d'Italie, d'Espagne, de Turquie et EAC-IBRO.

\section{Renseignements-inscriptions : Y. Ben-Ari-Inserm U. 129-123, boulevard de Port-Royal - 75014 Paris, France-Tél. : 0153737920 -Fax : 014634 1656. e-mail:neuromed@cochin.inserm.fr} Prix d'inscription : $700 \mathrm{FF}$ incluant participation, banquet et déjeuner

avec le soutien de Sanofi Winthrop -Cnrs -Conseil Général de l'Hérault-Laboratoires Cassenne-Ministère des Affaires Étrangères - Municipalité, ville de Montpellier-Novartis 